\title{
Evaluating Weight Status and Sex as Moderators of the Association of Serum Leptin with Bone Mineral Density in Children and Adolescents
}

\author{
Sara A. Armaiz-Flores ${ }^{a} \quad$ Nichole R. Kelly ${ }^{a}$ b $\quad$ Ovidiu A. Galescu ${ }^{a}$ \\ Andrew P. Demidowich ${ }^{a}$ Anne M. Altschula, c Sheila M. Brady ${ }^{a}$ Van S. Hubbard ${ }^{c}$ \\ Courtney K. Pickworth ${ }^{a}$ Marian Tanofsky-Kraffa, d Lauren B. Shomaker ${ }^{a, b}$ \\ James C. Reynolds ${ }^{\mathrm{e}}$ Jack A. Yanovski ${ }^{\mathrm{a}}$ \\ a Section on Growth and Obesity, Program in Developmental Endocrinology and Genetics, Eunice Kennedy Shriver \\ National Institute of Child Health and Human Development, National Institutes of Health (NIH), Bethesda, MD, USA; \\ ${ }^{b}$ Department of Human Development and Family Studies, Colorado State University, Fort Collins, CO, USA; ${ }^{\circ}$ Division of \\ Nutrition Research Coordination, National Institute of Diabetes and Digestive and Kidney Diseases, National Institutes \\ of Health (NIH), Bethesda, MD, USA; ${ }^{d}$ Department of Medical and Clinical Psychology, Uniformed Services University

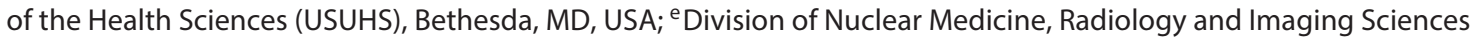 \\ Department, Hatfield Clinical Research Center, National Institutes of Health (NIH), Bethesda, MD, USA
}

\section{Keywords}

Leptin · Dual-energy X-ray absorptiometry · Pediatrics · Sex · Obesity · Bone mineral content · Bone area

\footnotetext{
Abstract

Background/Aims: Animal studies suggest that leptin may adversely affect bone mineral density (BMD). Clinical studies have yielded conflicting results. We therefore investigated associations between leptin and bone parameters in children. Methods: 830 healthy children (age $=11.4 \pm 3.1$ years; $75 \%$ female; BMI standard deviation score $[\mathrm{BMI}]=1.5 \pm 1.1$ ) had fasting serum leptin measured with ELISA and body composition by dual-energy X-ray absorptiometry. The main effects for leptin and BMIz plus leptin's interactions with sex and BMIz were examined using hierarchical linear regressions for appendicular, pelvis, and lumbar spine BMD as well as bone mineral content (BMC), and bone area (BA). Results: Accounting for demographic, pubertal development, and anthropometric variables, leptin was negatively and inde-
}

\section{KARGER}

(c) 2017 S. Karger AG, Basel

E-Mail karger@karger.com

www.karger.com/hrp pendently associated with lumbar spine $B M C$ and $B A$, pelvis $B A$, and leg BA ( $p<0.05$ for all). Sex, but not BMlz, moderated the associations of leptin with bone parameters. In boys, leptin was negatively correlated with leg and arm BMD, BMC at all bone sites, and BA at the subtotal and lumbar spine ( $p<0.01$ for all). In girls, leptin was positively correlated with leg and arm BMD ( $p<0.05$ for both). Conclusion: Independent of body size, leptin is negatively associated with bone measures; however, these associations are moderated by sex: boys, but not girls, have a negative independent association between leptin and BMD.

๑ 2017 S. Karger AG, Basel

\section{Introduction}

Recent data indicate that $17.4 \%$ of US children and $20.6 \%$ of adolescents are considered obese, with a body mass index (BMI) greater than or equal to the US Centers for Disease Control 95th percentile standard for age and
Jack A. Yanovski, MD, PhD

Section on Growth and Obesity, Division of Intramural Research, NICHD, NIH 10 Center Drive, Hatfield Clinical Research Center, Room 1E-3330, MSC 1103 Bethesda, MD 20892 (USA)

E-Mail jy15i@nih.gov 
sex [1]. Obesity affects many metabolic pathways, including glucose homeostasis, energy expenditure, growth, pubertal development, and bone mineralization [2]. Studies have shown that obesity is positively associated with bone mineral density (BMD) and bone mineral content (BMC) in adult and pediatric populations [3, 4]; however, obese children appear to be at an increased risk of fractures compared to nonobese children [5,6]. Childhood and adolescence are times of rapid growth and bone acquisition, influencing future fracture risk. It is therefore important to examine how obesity affects bone development in youth, and whether these associations differ among youth as the BMI standard deviation score (BMIz) increases.

Recent studies suggest that leptin, an adipocyte-derived hormone that is increased in obesity, may influence bone $[5,7]$. Leptin can cause bone resorption and osteoclast activation by inducing transcription of receptor activator of nuclear factor kappa-B ligand (RANKL) in osteoblasts via centrally mediated sympathetic pathways $[8$, 9]. Conversely, leptin may promote bone mineral acquisition locally through direct binding of leptin receptors expressed in primary osteoblasts $[10,11]$.

How these opposing effects relate to BMD in pediatric and adult cohorts is unclear. In studies involving adult females, higher leptin concentrations have been associated with higher BMD $[12,13]$. Moreover, leptin supplementation in adult women with hypothalamic amenorrhea decreases the RANKL to osteoprotegerin ratio, suggesting increased bone acquisition [14]. Conversely, in general pediatric and adult male samples, the associations between circulating leptin and bone measures have been negative [15-17] or nonsignificant [13, 18, 19]. Although these discrepancies could be the result of differences in study methodologies or inadequate sample size, they may also reflect sex- or developmentally related differences in leptin's effects on bone functioning.

To clarify the role of leptin in bone acquisition in obese youth, we evaluated the association between serum leptin and bone measures in a large sample of healthy children and adolescents enriched with overweight and obese participants, controlling for potential confounding factors, such as pubertal stage, height, and weight. Due to its use in clinical practice as a measure of bone mineral density, $\mathrm{BMD}$ was estimated from $\mathrm{BMC}$ and bone area (BA), but all 3 indices (BMD, BMC, and BA) were statistically analyzed.

Our primary aim was to examine the association between fasting serum leptin concentrations and bone parameters. Our secondary aims were to determine if the associations between leptin and bone measures differed based on BMIz and sex. We hypothesized that, after accounting for body adiposity and other relevant covariates (i.e., weight and fat mass percentage), leptin would demonstrate a negative association with bone measures [16]. We further hypothesized that this association would be moderated by both BMIz and sex: bone parameters in girls would have positive correlations with leptin; in contrast, among boys, bone parameters would have negative associations with leptin $[12,13,15,20]$.

In order to elucidate whether leptin contributes to the increased risk of fractures in obese children, BMIz was used as a grouping variable. No previous studies have examined BMIz as a moderator of the aforementioned associations. We hypothesized that individuals with lower BMIz might have a negative association between BMD and leptin, as suggested by previous pediatric studies, but individuals with a higher BMIz, given their increased fracture risk, would have an even stronger negative association between BMD and leptin.

\section{Subjects and Methods}

\section{Participants and Procedures}

A convenience sample of 830 pediatric participants was assembled from studies approved by the Institutional Review Board of the Eunice Kennedy Shriver National Institute of Child Health and Human Development (Clinicaltrials.gov identifiers: NCT01425905, NCT00001723, NCT00005669, NCT00001195, and NCT00001522). Studies involved the evaluation of growth and development of children and adolescents aged 5-18 years. Inclusion criteria required that participants were in good general health. Exclusion criteria included any major medical illness, a psychiatric condition likely to impede compliance to the study or the ability to give informed assent or consent, pregnancy, and the regular use of prescription medication affecting appetite or body weight. The occasional use of beta-agonist inhalers for asthma and nonsteroidal medications for pain was permitted. Children and adolescents provided written assent; parents or guardians provided written consent for participants. Only baseline data (i.e., prior to the initiation of any treatment) were included in the current analyses.

\section{Measures}

Body Composition and Bone Mineral Measurements

Height in centimeters to the nearest millimeter was measured in triplicate with a calibrated stadiometer (Holtain Ltd., Crymych, UK). Fasting weight was measured to the nearest $0.1 \mathrm{~kg}$ with a calibrated digital scale (Life Measurement Instruments, Concord, CA, USA). BMIz were calculated according to the standards of the Centers for Disease Control and Prevention [21]. Obesity was defined as a having a BMI $\geq 95$ th percentile for age and sex. Fat mass percentage, $\mathrm{BMD}, \mathrm{BMC}$, and $\mathrm{BA}$ were determined by dual-energy X-ray absorptiometry (Hologic QDR 2000, 4500A, or Discovery A instruments). Precision data between instruments, as calculated using phantom scans and human crossover studies, indicated ex- 
cellent precision with inter-instrument coefficients of variation of $0.8-4.3 \%$ for bone measures. Daily spine phantom measurements showed $<4 \%$ variability for each instrument within 6 -month windows. BMD, BMC, and BA estimates were analyzed for subtotal (whole body minus head), leg, arm, pelvis, and lumbar spine regions. Subtotal, rather than whole-body, measurements were used according to the recommendations of the International Society for Clinical Densitometry 2013 [22]. Segmental body measurements, such as lumbar spine, were obtained by extracting regional scans from whole-body scans, regions were set both by an automated computer program and visually confirmed by one of the authors (J.C.R.).

\section{Puberty}

Pubertal status was categorized by Tanner staging [23] via physical examination by an endocrinologist or nurse practitioner. Breast development was assessed in girls by inspection and palpation, and testicular volumes were measured in boys using orchidometry [24]. For girls, Tanner's 5 breast development stages were determined; for boys, testicular volumes were converted to stages (stage I: testes $\leq 3 \mathrm{~mL}$, stage II: $4-10 \mathrm{~mL}$, stage III: $10-16 \mathrm{~mL}$, stage IV: $17-24 \mathrm{~mL}$, stage $\mathrm{V}: \geq 25 \mathrm{~mL}$ ). The higher stage was assigned in case of discordance between size of breasts or testes [23, 25].

\section{Leptin}

Fasting plasma leptin was measured using commercially available assays (Linco, St. Charles, MO, USA, $n=467,20 \%$ males; or Mayo Medical Laboratories, Andover, MA, USA, $n=363$, 27\% males); neither assay differed significantly in the relationship between measured value and body fat mass [26]. The functional sensitivity for the assays was $0.4-0.5 \mathrm{ng} / \mathrm{mL}$; intra- and inter-assay variabilities were $<8$ and $<18 \%$, respectively.

\section{Statistical Analysis}

All statistical analyses were completed using PASWS statistics 18 (SPSS Statistics, IBM Corp.). Only participants with complete data were included for each analysis. Fasting leptin values were log transformed to correct for the exponential nature of the associations between leptin and BMD, BMC, and BA [7]. All other variables were normally distributed based on absolute skewness and kurtosis values ( $<3$ and 10, respectively). Fat mass percentage was arcsine transformed. Analysis of variance (ANOVA) was used to examine between-group differences in parametric variables among nonobese girls, obese girls, nonobese boys, and obese boys (age, $\mathrm{BMI}$, fat mass percentage, total body weight, height, fasting leptin, and the various bone measure sites). $\chi^{2}$ statistics were used to evaluate group differences for race $(0=$ non-Hispanic white, $1=$ other $)$ and weight status (obese vs. nonobese), and Kruskal-Wallis $\mathrm{H}$ test evaluated differences in pubertal stage by weight status [27]. Simple bivariate correlation analyses (i.e., unadjusted, no covariates) were run to confirm the established relationships between BMD, $\mathrm{BMC}$, and BA and leptin.

To evaluate our primary aim of examining the associations between fasting leptin concentrations and bone measures, a series of hierarchical multiple regression models were evaluated. Covariates entered in the first level of the model were age, sex, race, pubertal status, height, weight, and fat mass. The mean serum leptin level was significantly higher among participants measured using the Mayo versus Linco assay $(25.8 \pm 48.8 \mathrm{ng} / \mathrm{mL}$ vs. $17.5 \pm$ $56.5 \mathrm{ng} / \mathrm{mL}, p=0.02)$; therefore, leptin assay type was also consid- ered as a covariate. As including this variable did not change the direction or significance of any result, it was dropped from the final models presented. Leptin ( $\mathrm{ng} / \mathrm{mL}, \log$ transformed) was added to the second level of each model. One model was conducted for each dependent variable, including BMD $\left(\mathrm{g} / \mathrm{cm}^{2}\right), \mathrm{BMC}(\mathrm{g})$, and BA $\left(\mathrm{cm}^{2}\right)$ for each bone site. To determine if BMIz or sex moderated the effects of leptin on bone parameters, hierarchical linear regression models were used for each of the bone sites measured. The first level included age, sex, race, pubertal status, height, weight, and fat mass; these covariates were included irrespective of their statistical significance in the model. The second level included leptin ( $\mathrm{ng} / \mathrm{mL}$, log transformed), BMIz, and sex. The third level included the product terms between leptin and BMIz and between leptin and sex. Leptin and BMIz were centered based on the grand mean prior to being entered into each of the models and the respective interaction terms. To determine if sex or Tanner stage moderated the effects of leptin on bone parameters, hierarchical linear regression models were used for each of the bone sites measured. Models were constructed as mentioned previously with the exception of the third level, including the product term of sex $\times$ leptin, sex $\times$ Tanner stage, and leptin $\times$ Tanner stage. A fourth level included the product term of leptin $\times$ sex $\times$ Tanner stage. For models with significant interaction terms, additional regression models were conducted to evaluate the main effects. The Benjamini-Hochberg procedure was used to correct for multiple comparisons [28].

\section{Results}

\section{Sample Characteristics}

Data were analyzed from 830 participants $(74.9 \%$ female, mean age $=11.4 \pm 3.1$ years, $56.5 \%$ obese). Tanner stage distribution for the cohort was as follows: Tanner I, $34.4 \%$ (26.2\% of females; $59.7 \%$ of males); Tanner II, $19.7 \%$ (20.2\% of females; $17.9 \%$ of males); Tanner III, $13.7 \%$ ( $15.7 \%$ of females; $8.5 \%$ of males); Tanner IV, $8.8 \%$ (10.5\% of females; $3.5 \%$ of males); and Tanner V, $23.2 \%$ (27.4\% of females; $10.4 \%$ of males). Obese girls were older, had a higher pubertal stage, were taller, and were heavier compared to nonobese girls, nonobese boys, and obese boys ( $p \leq 0.001$ for all). Nonobese girls had a higher fat mass percentage and serum leptin compared to nonobese boys ( $p \leq 0.001$ for all), but they did not differ significantly in any other variable (Table 1). Obese girls did not differ significantly from nonobese boys and obese boys in lumbar spine BA; however, they did differ significantly in all other variables ( $p \leq 0.01$ for all). All measures of bone parameters were higher for obese girls compared to nonobese girls ( $p \leq 0.001$ for all). Obese boys did not differ significantly in age compared to nonobese boys; however, they did differ from nonobese boys in all other variables (Table $1 ; p \leq 0.05$ for all). 
Table 1. Descriptive statistics for the total sample group and comparisons based on weight status and sex

\begin{tabular}{|c|c|c|c|c|c|}
\hline Age, years & $11.4 \pm 3.1$ & $10.6 \pm 2.9^{\mathrm{a}}$ & $12.3 \pm 2.9^{\mathrm{a}, \mathrm{b}}$ & $10.3 \pm 3.2^{\mathrm{b}}$ & $11.2 \pm 3.1^{\mathrm{b}}$ \\
\hline $\begin{array}{l}\text { Median pubertal development } \\
\text { stage (range) }\end{array}$ & $2.0(1-5)$ & $2.0(1-5)^{\mathrm{a}, \mathrm{b}, \mathrm{c}}$ & $3.0(1-5)^{a, b, c}$ & $1.0(1-5)^{\mathrm{a}, \mathrm{b}, \mathrm{c}}$ & $2.0(1-5)^{\mathrm{a}, \mathrm{b}, \mathrm{c}}$ \\
\hline Non-Hispanic white race, $\%$ & 47.2 & 46.2 & 40.5 & 63.0 & 56.0 \\
\hline Height, $\mathrm{cm}$ & $149.2 \pm 16.9$ & $141.8 \pm 16.0^{\mathrm{a}}$ & $155.6 \pm 13.4^{\mathrm{a}, \mathrm{b}}$ & $142.1 \pm 19.1^{\mathrm{b}, \mathrm{c}}$ & $152.1 \pm 17.1^{\mathrm{a}, \mathrm{c}}$ \\
\hline BMI & $27.4 \pm 9.6$ & $19.2 \pm 3.9^{\mathrm{a}}$ & $34.4 \pm 6.7^{\mathrm{a}, \mathrm{b}}$ & $18.1 \pm 2.6^{\mathrm{b}, \mathrm{c}}$ & $32.9 \pm 8.7^{\mathrm{a}, \mathrm{c}}$ \\
\hline BMIz & $1.53 \pm 1.14$ & $0.4 \pm 0.9^{a}$ & $2.4 \pm 0.3^{\mathrm{a}, \mathrm{b}}$ & $0.4 \pm 0.7^{\mathrm{b}, \mathrm{c}}$ & $2.4 \pm 0.4^{\mathrm{a}, \mathrm{c}}$ \\
\hline Fat mass, \% & $35.1 \pm 11.9$ & $26.8 \pm 8.1^{\mathrm{a}, \mathrm{b}, \mathrm{c}}$ & $44.1 \pm 4.8^{\mathrm{a}, \mathrm{b}, \mathrm{c}}$ & $18.9 \pm 6.2^{\mathrm{a}, \mathrm{b}, \mathrm{c}}$ & $41.1 \pm 7.9^{\mathrm{a}, \mathrm{b}, \mathrm{c}}$ \\
\hline Leptin, ng/dL & $21.8 \pm 19.7$ & $9.5 \pm 11.4^{\mathrm{a}, \mathrm{b}, \mathrm{c}}$ & $34.7 \pm 17.7^{\mathrm{a}, \mathrm{b}, \mathrm{c}}$ & $3.03 \pm 2.2^{\mathrm{a}, \mathrm{b}, \mathrm{c}}$ & $26.0 \pm 18.0^{\mathrm{a}, \mathrm{b}, \mathrm{c}}$ \\
\hline Subtotal BMD, $\mathrm{g} / \mathrm{cm}^{2}$ & $0.8 \pm 0.2$ & $0.8 \pm 0.2^{\mathrm{a}, \mathrm{b}}$ & $0.9 \pm 0.2^{\mathrm{a}, \mathrm{b}}$ & $0.3 \pm 0.2^{\mathrm{b}, \mathrm{c}}$ & $0.8 \pm 0.1^{\mathrm{a}, \mathrm{b}, \mathrm{c}}$ \\
\hline Pelvis $\mathrm{BMD}, \mathrm{g} / \mathrm{cm}^{2}$ & $1.0 \pm 0.3$ & $0.9 \pm 0.2^{\mathrm{a}, \mathrm{b}}$ & $1.1 \pm 0.2^{\mathrm{a}, \mathrm{b}, \mathrm{c}}$ & $0.9 \pm 0.2^{b, c}$ & $1.0 \pm 0.2^{\mathrm{a}, \mathrm{b}, \mathrm{c}}$ \\
\hline Pelvis BMC, $\mathrm{g}$ & $167.2 \pm 94.4$ & $125.3 \pm 72.1^{\mathrm{a}, \mathrm{b}}$ & $201.5 \pm 94.4^{\mathrm{a}, \mathrm{b}, \mathrm{c}}$ & $138.8 \pm 95.3^{b, c}$ & $171.1 \pm 85.7^{\mathrm{a}, \mathrm{b}, \mathrm{c}}$ \\
\hline Lumbar BMC, g & $29.6 \pm 14.8$ & $24.9 \pm 12.5^{\mathrm{a}, \mathrm{b}}$ & $33.6 \pm 14.6^{\mathrm{a}, \mathrm{b}, \mathrm{c}}$ & $27.5 \pm 16.5^{b, c}$ & $29.0 \pm 13.7^{\mathrm{b}}$ \\
\hline Leg BMC, g & $313.9 \pm 147.9$ & $232.7 \pm 123.7^{\mathrm{a}, \mathrm{b}}$ & $387.1 \pm 122.8^{\mathrm{a}, \mathrm{b}, \mathrm{c}}$ & $223.3 \pm 141.1^{b, c}$ & $343.4 \pm 135.4^{\mathrm{a}, \mathrm{b}, \mathrm{c}}$ \\
\hline Arm BMC, g & $106.7 \pm 55.4$ & $76.1 \pm 46.2^{\mathrm{a}, \mathrm{b}}$ & $132.8 \pm 46.8^{\mathrm{a}, \mathrm{b}, \mathrm{c}}$ & $75.9 \pm 54.2^{\mathrm{b}, \mathrm{c}}$ & $118.6 \pm 50.0^{\mathrm{a}, \mathrm{c}}$ \\
\hline Subtotal BA, $\mathrm{cm}^{2}$ & $1,456.5 \pm 446.4$ & $1,182.0 \pm 400.8^{\mathrm{a}, \mathrm{b}}$ & $1,682.7 \pm 327.0^{a, b, c}$ & $1,174.1 \pm 427.4^{\mathrm{b}, \mathrm{c}}$ & $1,611.1 \pm 399.6^{\mathrm{a}, \mathrm{c}}$ \\
\hline Pelvis $\mathrm{BA}, \mathrm{cm}^{2}$ & $149.4 \pm 49.2$ & $132.6 \pm 45.3^{\mathrm{a}, \mathrm{b}}$ & $159.3 \pm 46.9^{a, b, c}$ & $146.2 \pm 94.1^{b, c}$ & $156.0 \pm 45.4^{\mathrm{a}}$ \\
\hline Lumbar BA, $\mathrm{cm}^{2}$ & $35.5 \pm 10.1$ & $33.1 \pm 9.3^{\mathrm{a}}$ & $36.8 \pm 9.4^{\mathrm{a}}$ & $35.6 \pm 11.5$ & $35.8 \pm 10.4$ \\
\hline Leg $\mathrm{BA}, \mathrm{cm}^{2}$ & $300.4 \pm 92.1$ & $246.0 \pm 83.0^{\mathrm{a}, \mathrm{b}}$ & $345.0 \pm 64.9^{\mathrm{a}, \mathrm{b}, \mathrm{c}}$ & $240.6 \pm 94.1^{\mathrm{b}, \mathrm{c}}$ & $337.1 \pm 85.8^{\mathrm{a}, \mathrm{c}}$ \\
\hline Arm BA, $\mathrm{cm}^{2}$ & $153.1 \pm 56.2$ & $116.5 \pm 47.8^{\mathrm{a}, \mathrm{b}}$ & $181.0 \pm 40.4^{\mathrm{a}, \mathrm{b}, \mathrm{c}}$ & $117.5 \pm 54.7^{\mathrm{b}, \mathrm{c}}$ & $179.6 \pm 52.5^{\text {a, c }}$ \\
\hline
\end{tabular}

Data are reported as mean $\pm S D$, unless otherwise noted. Each superscript represents a statistically significant difference between the means within groups with the same subscript. BMI, body mass index; BMIz, BMI standard deviation score; BMD, bone mineral density; $\mathrm{BMC}$, bone mineral content; BA, bone area.

\section{Unadjusted and Adjusted Associations between Leptin and Body Composition}

As expected, simple correlation analysis found strong positive associations between serum leptin and percentage fat mass $(r=0.72, p<0.0001)$, as well as with subtotal $\operatorname{BMD}(r=0.66, p<0.0001), \mathrm{BMC}(r=0.32, p<0.0001)$, and BA $(r=0.40, p<0.0001)$. In the multivariate models adjusting for covariates including weight $(p<0.001)$ and fat mass $(p<0.001)$, there were negative associations between leptin and lumbar spine BMC $(b=-0.133, p=$ $0.006)$, pelvis $B A(b=-0.207, p \leq 0.001)$, lumbar spine $B A$ $(\mathrm{b}=-0.190, p=0.001)$, and leg BA $(\mathrm{b}=0.137, p=0.02$; Fig. 1; Table 2; online suppl. Table 1; for all online suppl. material, see www.karger.com/doi/10.1159/000459623). However, we found no significant independent associations between leptin and BMD (Table 2) at any site.
BMIz and Sex as Moderators of the Association between Leptin and Bone Parameters

There was no significant interaction between BMIz and leptin ( $p=0.1$ to 0.9 for all) at any examined site (online suppl. Table 2). As expected, simple correlation analysis found positive associations between serum leptin and subtotal BMD $(r=0.06, p=0.001)$, BMC $(r=0.11, p<$ $0.0001)$, and BA $(r=0.20, p<0.0001)$ in boys; similar positive correlations were found in girls for subtotal BMD $(r=0.34, p<0.0001)$, BMC $(r=0.43, p<0.0001)$, and BA $(r=0.50, p<0.0001)$. Significant interactions between sex and leptin (online suppl. Table 3 ) were found for subtotal $\operatorname{BMD}(p<0.003)$, lumbar spine BMD $(p=0.005)$, leg $\mathrm{BMD}(p=0.001)$, arm BMD $(p=0.001)$, subtotal BMC $(p<0.001)$, pelvis $\mathrm{BMC}(p=0.03)$, lumbar spine BMC $(p=0.002)$, leg BMC $(p=0.01)$, arm BMC $(p=0.04)$, subtotal BA $(p=0.005)$, and lumbar spine BA $(p=0.04)$. In 


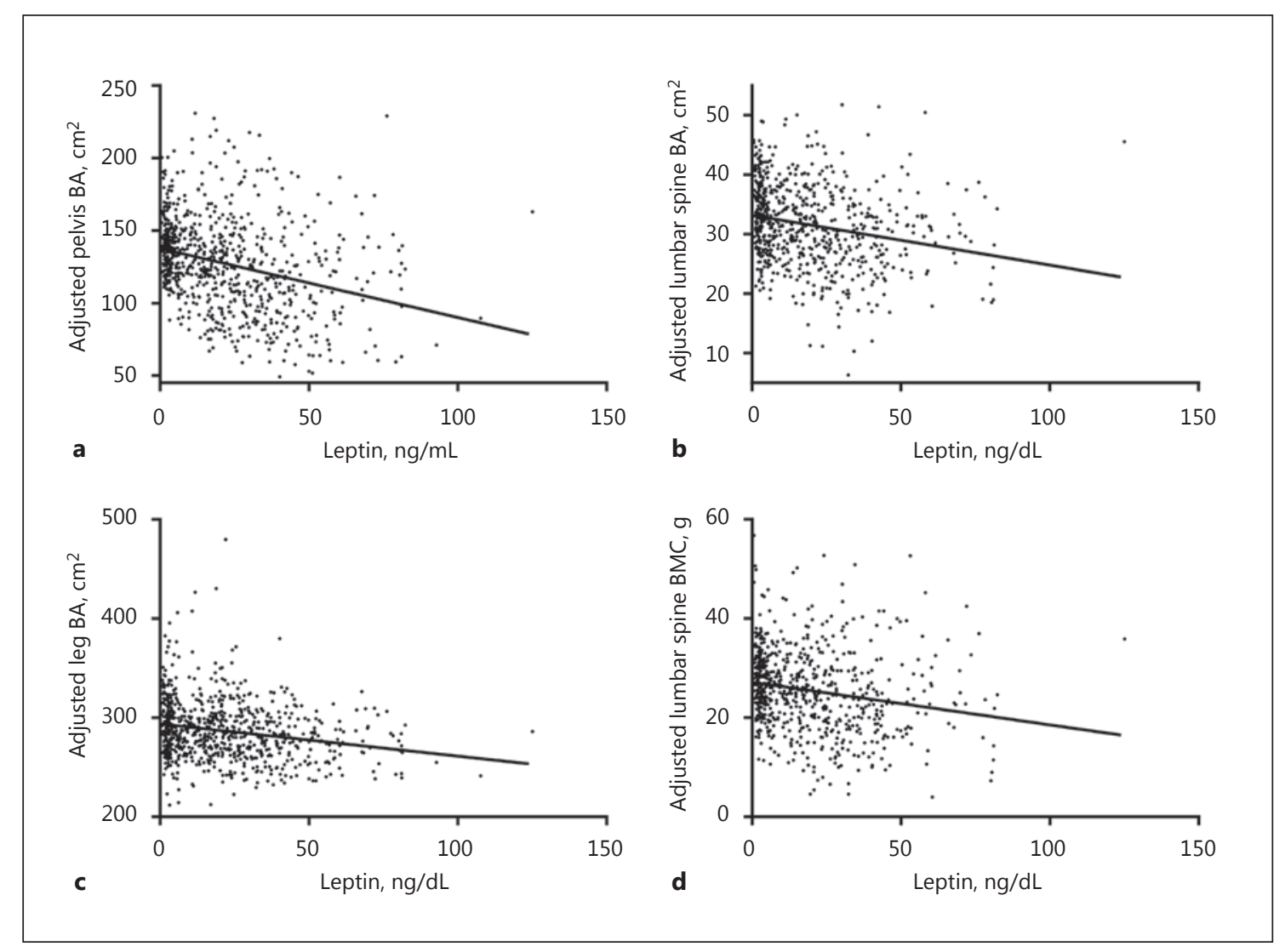

Fig. 1. In children and adolescents, leptin was negatively associated with pelvis BA $\left(r^{2}=0.088, p<0.001\right)(\mathbf{a})$, lumbar spine BA $\left(r^{2}=0.05, p<0.001\right)(\mathbf{b})$, leg BA $\left(r^{2}=0.045, p<0.001\right)(\mathbf{c})$, and lumbar spine BMC $\left(r^{2}=0.036\right.$, $p<0.001)(\mathbf{d})$.

analyses restricted to boys (Table 3 ), leptin was negative$l y$ associated with BMD in the appendicular skeleton (leg: $p=0.02$; arm: $p=0.02$ ); among girls (Table 3), leptin was positively associated with leg $\operatorname{BMD}(p=0.002)$ and arm $\operatorname{BMD}(p=0.002)$. In boys (Table 3$)$, leptin had a negative association with BMC at all examined sites ( $p \leq 0.003$ for all). In girls (Table 3 ), leptin was not significantly associated with any BMC measure $(p=0.1-0.9)$. Leptin was negatively associated with subtotal BA $(p=0.01)$ and lumbar BA ( $p \leq 0.001$ ) in boys (Table 3 ), but was not significantly associated with any BA measure in girls (Table $3 ; p=0.1-0.9)$. Leptin contributed the greatest amount of variance in the models for lumbar BMC and BA in boys $\left(\Delta R^{2}=0.023\right.$ and 0.032 , respectively). No significant triple interaction terms were found between sex, leptin, and Tanner stage.

\section{Discussion}

Despite the positive association between BMD and BMIz, studies have found that obese children have an increased risk of fractures and consequently undergo more orthopedic interventions $[5,6]$. It is important to therefore explore whether the altered metabolic environment in obesity contributes to this association. After accounting for body mass and other relevant covariates, we found a negative correlation between leptin and lumbar BMC, as well as pelvis, lumbar, and leg BA. These results are consistent with a recent study reporting that leptin had a negative correlation with tibial trabecular thickness in a sample of mostly obese children [20]. This association suggests that the central catabolic effects of leptin that have been elucidated in murine models [8] may also play a role in pediatric bone development and mineralization. Leptin appears to promote osteoclastogenesis by 
Table 2. Multiple hierarchical linear regressions examining the association between leptin and bone mineral density and content

\begin{tabular}{|c|c|c|c|c|c|c|c|c|}
\hline $\begin{array}{l}\text { Dependent } \\
\text { variables }\end{array}$ & Levels & $\begin{array}{l}\text { Variable } \\
\text { entered }\end{array}$ & $\begin{array}{l}\beta \\
\text { (unstandardized) }\end{array}$ & SE & $\begin{array}{l}\text { b } \\
\text { (standardized) }\end{array}$ & $p$ & $R^{2}$ & $\Delta R^{2}$ \\
\hline \multirow[t]{2}{*}{$\begin{array}{l}\text { Subtotal BMD } \\
\left(\mathrm{g} / \mathrm{cm}^{2)}\right.\end{array}$} & 1 & $\begin{array}{l}\text { age } \\
\text { sex } \\
\text { race } \\
\text { pubertal stage } \\
\text { height } \\
\text { weight } \\
\text { fat mass \% }\end{array}$ & $\begin{array}{r}0.014 \\
-0.003 \\
0.038 \\
0.035 \\
0.002 \\
0.001 \\
0.013 \\
\end{array}$ & $\begin{array}{l}0.002 \\
0.008 \\
0.006 \\
0.004 \\
0.000 \\
0.000 \\
0.039 \\
\end{array}$ & $\begin{array}{l}0.231^{* *} \\
-0.008 \\
0.101^{* *} \\
0.290^{* *} \\
0.218^{* *} \\
0.203^{* *} \\
0.009 \\
\end{array}$ & $\begin{array}{c}<0.001 \\
0.7 \\
<0.001 \\
<0.001 \\
<0.001 \\
<0.001 \\
0.7 \\
\end{array}$ & 0.791 & - \\
\hline & 2 & leptin & 0.020 & 0.015 & 0.056 & 0.2 & - & - \\
\hline \multirow[t]{2}{*}{$\begin{array}{l}\text { Pelvis BMD } \\
\left(\mathrm{g} / \mathrm{cm}^{2}\right)\end{array}$} & 1 & $\begin{array}{l}\text { age } \\
\text { sex } \\
\text { race } \\
\text { pubertal stage } \\
\text { height } \\
\text { weight } \\
\text { fat mass \% }\end{array}$ & $\begin{array}{r}0.011 \\
-0.004 \\
0.012 \\
0.038 \\
0.002 \\
0.005 \\
-0.251\end{array}$ & $\begin{array}{l}0.003 \\
0.011 \\
0.009 \\
0.005 \\
0.001 \\
0.000 \\
0.052\end{array}$ & $\begin{array}{c}0.137^{* *} \\
-0.006 \\
0.024 \\
0.230^{* *} \\
0.113^{* *} \\
0.567^{* *} \\
-0.128^{* *}\end{array}$ & $\begin{array}{c}<0.001 \\
0.7 \\
0.2 \\
<0.001 \\
0.004 \\
<0.001 \\
<0.001\end{array}$ & 0.792 & - \\
\hline & $\overline{2}$ & leptin & 0.005 & 0.020 & 0.010 & 0.8 & - & - \\
\hline \multirow[t]{2}{*}{$\begin{array}{l}\text { Lumbar spine } \\
\operatorname{BMD}\left(\mathrm{g} / \mathrm{cm}^{2}\right)\end{array}$} & 1 & $\begin{array}{l}\text { age } \\
\text { sex } \\
\text { race } \\
\text { pubertal stage } \\
\text { height } \\
\text { weight } \\
\text { fat mass \% }\end{array}$ & $\begin{array}{r}0.014 \\
-0.023 \\
0.028 \\
0.049 \\
0.000 \\
0.003 \\
-0.256 \\
\end{array}$ & $\begin{array}{l}0.003 \\
0.009 \\
0.008 \\
0.004 \\
0.001 \\
0.000 \\
0.047 \\
\end{array}$ & $\begin{array}{c}0.212^{* *} \\
-0.053^{*} \\
0.071^{* *} \\
0.356^{* *} \\
0.030 \\
0.421^{* *} \\
-0.171^{* *} \\
\end{array}$ & $\begin{array}{c}<0.001 \\
0.01 \\
<0.001 \\
<0.001 \\
0.5 \\
<0.001 \\
<0.001 \\
\end{array}$ & 0.748 & - \\
\hline & 2 & leptin & -0.005 & 0.018 & -0.013 & 0.8 & - & - \\
\hline \multirow[t]{2}{*}{$\begin{array}{l}\text { Leg BMD } \\
\left(\mathrm{g} / \mathrm{cm}^{2}\right)\end{array}$} & 1 & $\begin{array}{l}\text { age } \\
\text { sex } \\
\text { race } \\
\text { pubertal stage } \\
\text { height } \\
\text { weight } \\
\text { fat mass \% }\end{array}$ & $\begin{array}{l}0.015 \\
0.001 \\
0.055 \\
0.033 \\
0.003 \\
0.002 \\
0.088 \\
\end{array}$ & $\begin{array}{l}0.003 \\
0.009 \\
0.007 \\
0.004 \\
0.000 \\
0.000 \\
0.042 \\
\end{array}$ & $\begin{array}{l}0.209^{* *} \\
0.001 \\
0.126^{* *} \\
0.239^{* *} \\
0.230^{* *} \\
0.247^{* *} \\
0.053^{*}\end{array}$ & $\begin{array}{c}<0.001 \\
0.9 \\
<0.001 \\
<0.001 \\
<0.001 \\
<0.001 \\
0.04 \\
\end{array}$ & 0.814 & - \\
\hline & 2 & leptin & 0.025 & 0.016 & 0.059 & 0.1 & - & - \\
\hline \multirow[t]{2}{*}{$\begin{array}{l}\text { Arm BMD } \\
\left(\mathrm{g} / \mathrm{cm}^{2}\right)\end{array}$} & 1 & $\begin{array}{l}\text { age } \\
\text { sex } \\
\text { race } \\
\text { pubertal stage } \\
\text { height } \\
\text { weight } \\
\text { fat mass \% }\end{array}$ & $\begin{array}{r}0.013 \\
0.000 \\
0.025 \\
0.027 \\
0.001 \\
0.001 \\
-0.080 \\
\end{array}$ & $\begin{array}{l}0.002 \\
0.007 \\
0.006 \\
0.003 \\
0.000 \\
0.000 \\
0.033 \\
\end{array}$ & $\begin{array}{c}0.290^{* *} \\
-0.001 \\
0.088^{* *} \\
0.292^{* *} \\
0.107^{*} \\
0.261^{* *} \\
-0.073^{*}\end{array}$ & $\begin{array}{c}<0.001 \\
0.9 \\
<0.001 \\
<0.001 \\
0.02 \\
<0.001 \\
0.017 \\
\end{array}$ & 0.726 & - \\
\hline & 2 & leptin & 0.025 & 0.013 & 0.088 & 0.053 & - & - \\
\hline \multirow[t]{2}{*}{$\begin{array}{l}\text { Subtotal BMC } \\
(\mathrm{g})\end{array}$} & 1 & $\begin{array}{l}\text { age } \\
\text { sex } \\
\text { race } \\
\text { pubertal stage } \\
\text { height } \\
\text { weight } \\
\text { fat mass \% }\end{array}$ & $\begin{array}{r}20.219 \\
27.167 \\
71.357 \\
90.935 \\
13.134 \\
7.073 \\
4.516 \\
\end{array}$ & $\begin{array}{r}5.635 \\
19.105 \\
15.216 \\
8.384 \\
1.053 \\
0.574 \\
91.401 \\
\end{array}$ & $\begin{array}{l}0.099^{* *} \\
0.018 \\
0.056^{* *} \\
0.226^{* *} \\
0.349^{* *} \\
0.354^{* *} \\
0.001 \\
\end{array}$ & $\begin{array}{l}<0.001 \\
0.2 \\
<0.001 \\
<0.001 \\
<0.001 \\
<0.001 \\
0.9\end{array}$ & 0.895 & - \\
\hline & 2 & leptin & -13.956 & 34.563 & -0.011 & 0.7 & - & - \\
\hline
\end{tabular}


Table 2 (continued)

\begin{tabular}{|c|c|c|c|c|c|c|c|c|}
\hline $\begin{array}{l}\text { Dependent } \\
\text { variables }\end{array}$ & Levels & $\begin{array}{l}\text { Variable } \\
\text { entered }\end{array}$ & $\begin{array}{l}\beta \\
\text { (unstandardized) }\end{array}$ & SE & $\begin{array}{l}\text { b } \\
\text { (standardized) }\end{array}$ & $p$ & $R^{2}$ & $\Delta R^{2}$ \\
\hline \multirow{8}{*}{$\begin{array}{l}\text { Pelvis BMC } \\
\text { (g) }\end{array}$} & \multirow[t]{7}{*}{1} & age & 2.883 & 1.294 & $0.095^{*}$ & 0.03 & \multirow[t]{7}{*}{0.749} & \multirow[t]{7}{*}{-} \\
\hline & & sex & 4.065 & 4.391 & 0.019 & 0.4 & & \\
\hline & & race & -3.130 & 3.499 & -0.017 & 0.4 & & \\
\hline & & pubertal stage & 16.493 & 1.927 & $0.276^{* *}$ & $<0.001$ & & \\
\hline & & height & 1.304 & 0.241 & $0.233^{* *}$ & $<0.001$ & & \\
\hline & & weight & 1.427 & 0.131 & $0.483^{* *}$ & $<0.001$ & & \\
\hline & & fat mass $\%$ & -171.839 & 20.984 & $-0.240^{* *}$ & $<0.001$ & & \\
\hline & 2 & leptin & -13.102 & 7.929 & -0.072 & 0.1 & - & - \\
\hline \multirow{8}{*}{$\begin{array}{l}\text { Lumbar spine } \\
\text { BMC (g) }\end{array}$} & \multirow[t]{7}{*}{1} & age & 0.358 & 0.242 & 0.073 & 0.1 & \multirow[t]{7}{*}{0.713} & \multirow[t]{7}{*}{-} \\
\hline & & sex & -0.007 & 0.740 & 0.000 & 0.9 & & \\
\hline & & race & -0.546 & 0.622 & -0.019 & 0.4 & & \\
\hline & & pubertal stage & 3.754 & 0.349 & $0.373^{* *}$ & $<0.001$ & & \\
\hline & & height & 0.382 & 0.044 & $0.437^{* *}$ & $<0.001$ & & \\
\hline & & weight & 0.048 & 0.024 & $0.105^{*}$ & 0.05 & & \\
\hline & & fat mass $\%$ & -18.762 & 3.710 & $-0.172^{* *}$ & 0.000 & & \\
\hline & 2 & leptin & -3.830 & 1.378 & $-0.133^{*}$ & 0.006 & 0.716 & 0.003 \\
\hline \multirow{8}{*}{$\begin{array}{l}\text { Leg BMC } \\
(\mathrm{g})\end{array}$} & \multirow[t]{7}{*}{1} & age & 1.636 & 1.313 & 0.034 & 0.2 & \multirow[t]{7}{*}{0.895} & \multirow[t]{7}{*}{-} \\
\hline & & $\operatorname{sex}$ & 7.343 & 4.462 & 0.021 & 0.1 & & \\
\hline & & race & 24.558 & 3.554 & 0.083 & $<0.001$ & & \\
\hline & & pubertal stage & 14.043 & 1.958 & 0.149 & $<0.001$ & & \\
\hline & & height & 4.283 & 0.245 & 0.486 & $<0.001$ & & \\
\hline & & weight & 1.443 & 0.133 & 0.310 & $<0.001$ & & \\
\hline & & fat mass $\%$ & 49.492 & 21.314 & 0.044 & 0.020 & & \\
\hline & 2 & leptin & -10.585 & 8.061 & -0.037 & 0.2 & - & - \\
\hline \multirow{8}{*}{$\begin{array}{l}\text { Arm BMC } \\
(\mathrm{g})\end{array}$} & \multirow[t]{7}{*}{1} & age & 2.411 & 0.504 & $0.135^{* *}$ & $<0.001$ & \multirow[t]{7}{*}{0.890} & \multirow[t]{7}{*}{-} \\
\hline & & sex & 4.512 & 1.713 & $0.035^{*}$ & 0.01 & & \\
\hline & & race & 6.754 & 1.364 & $0.061^{* *}$ & $<0.001$ & & \\
\hline & & pubertal stage & 6.501 & 0.752 & $0.185^{* *}$ & $<0.001$ & & \\
\hline & & height & 0.923 & 0.094 & $0.280^{* *}$ & $<0.001$ & & \\
\hline & & weight & 0.789 & 0.051 & $0.453^{* *}$ & $<0.001$ & & \\
\hline & & fat mass $\%$ & -21.027 & 8.181 & $-0.050^{*}$ & 0.010 & & \\
\hline & 2 & leptin & -0.551 & 3.097 & -0.005 & 0.9 & - & - \\
\hline
\end{tabular}

Standardized $b$, the degree by which the dependent variable increases or decreases for every SE (standard deviation) increment; $R^{2}$, proportion of variability in the dependent variable accounted for by model; $\Delta R^{2}$, change in variability in the dependent variable accounted for by the additional models; BMD, bone mineral density; $\mathrm{BMC}$, bone mineral content; BA, bone area. ${ }^{* *} p<0.0001 ;{ }^{*} p<0.05$.

stimulating the sympathetic system to cause $\beta_{2}$ adrenergic receptor activation in bone $[8,9]$. Leptin may also be acting as a proinflammatory molecule inducing bone resorption $[29,30]$.

We observed that the inverse relationship between leptin and bone was greatest in the axial skeleton, which is composed mostly of trabecular bone. Trabecular bone formation occurs through endochondral ossification [31], while cortical bone is the product of trabecular re- modeling and periosteal expansion [32]. Both types of bone are influenced, albeit differently, by hormonal and environmental factors. Studies have found increases in cortical but not in trabecular bone due to increased exercise and growth hormone supplementation in murine models and human studies [33, 34], while pulsatile parathyroid hormone [35] seems to stimulate trabecular bone growth. It is possible that trabecular bone could be preferentially affected by the catabolic effects of leptin, while 
Table 3. Associations between leptin and bone measures in analyses restricted to boys and restricted to girls for variables where sex significantly moderated the association between leptin and bone mineral measures

\begin{tabular}{|c|c|c|c|c|c|c|}
\hline \multirow[t]{2}{*}{ Dependent variable } & \multicolumn{3}{|l|}{ Boys } & \multicolumn{3}{|l|}{ Girls } \\
\hline & $\Delta R^{2}$ & $\begin{array}{l}\text { Leptin (ng/dL) } \\
\beta\end{array}$ & $p$ & $\Delta \mathrm{R}^{2}$ & $\begin{array}{l}\text { Leptin (ng/dL) } \\
\beta\end{array}$ & $p$ \\
\hline Subtotal BMD $\left(\mathrm{g} / \mathrm{cm}^{2}\right)$ & 0.005 & -0.176 & 0.012 & 0.002 & 0.090 & 0.1 \\
\hline Lumbar BMD $\left(\mathrm{g} / \mathrm{cm}^{2}\right)$ & 0.002 & -0.104 & 0.3 & - & -0.005 & 0.8 \\
\hline $\operatorname{Leg} \operatorname{BMD}\left(\mathrm{g} / \mathrm{cm}^{2}\right)$ & 0.005 & -0.173 & 0.02 & 0.002 & 0.107 & 0.002 \\
\hline $\operatorname{Arm} \operatorname{BMD}\left(\mathrm{g} / \mathrm{cm}^{2}\right)$ & 0.007 & -0.206 & 0.02 & 0.004 & 0.139 & 0.002 \\
\hline Subtotal BMC (g) & 0.008 & -0.222 & $<0.001$ & - & 0.042 & 0.1 \\
\hline Pelvis BMC (g) & 0.002 & -0.211 & 0.003 & - & -0.014 & 0.8 \\
\hline Lumbar BMC (g) & 0.023 & -0.374 & $<0.001$ & - & -0.039 & 0.52 \\
\hline Leg BMC (g) & 0.010 & -0.242 & $<0.001$ & - & 0.034 & 0.1 \\
\hline Subtotal BA $\left(\mathrm{cm}^{2}\right)$ & 0.003 & -0.133 & 0.01 & - & -0.328 & 0.9 \\
\hline Lumbar BA $\left(\mathrm{cm}^{2}\right)$ & 0.032 & -0.446 & $<0.001$ & 0.001 & -0.080 & 0.2 \\
\hline
\end{tabular}

All $p$ values reported after using the Benjamini-Hochberg procedure to correct for multiple comparisons. Reporting betas for models after correcting for the following covariates: age, sex, race, pubertal status, height, weight, and fat mass. BMD, bone mineral density; BMC, bone mineral content; BA, bone area.

cortical bone acquisition could be controlled more by other hormonal factors.

We also found that, in analyses that first accounted for fat and lean mass, BMIz did not further moderate the relationship of leptin with bone measurements. These data suggest that leptin's associations with bone parameters do not change among youth as BMIz increases. Therefore, leptin does not have a greater impact on bone among obese versus nonobese children, and the hyperleptinemic state of obesity is most likely not contributing significantly to obese children's increased incidence of fractures; the greater force incident upon bone from a heavier corpus may be the more salient factor in worsening fracture rates among obese children [36]. However, sex did alter the directionality of the relationship between leptin and bone variables (Fig. 2). We found that for girls, leptin had a positive association with leg and arm BMD, whereas for boys, leptin had a negative correlation with BMD at these sites. Similar differences in leptin's associations with bone measures have been reported in studies involving nonobese female adolescents and young men $[15,19]$. Although leptin increases at a different rate in boys and girls throughout puberty, these changes are most likely due to gains in fat mass percentage; because we accounted for this variable in all of our models, our results should not be due to changing leptin concentrations during puberty. However, in order to examine whether puberty did modulate the directionality of our models, triple interaction terms were constructed, and puberty was found not to moderate the interaction between sex and leptin and their relationship with the bone parameters measured. The observed sexual dimorphisms suggest that girls may be protected from the effects of hyperleptinemia, perhaps due to their higher estrogen concentrations, even during childhood [37]. Leptin has been shown to alter estrogen receptor (ER) expression by upregulating ER $\alpha$ and ER $\beta$ protein levels in the chondrocytes of the growth plate [38]. Ohlsson et al. [39] found that ERa knockout mice have increased BMD and peripheral leptin concentrations. They proposed that lower central estrogen concentrations decrease central leptin activity and increase white adipose tissue production of peripheral leptin. [39]. Therefore, higher estrogen levels in obese (vs. nonobese) males could be activating central leptin pathways. Because estrogen levels in premenopausal obese females do not vary significantly from those in nonobese females [40], obese females could be protected from central leptin pathway activation. It is possible that the high level of cross-talk between estrogen and leptin receptors could explain the sexual dimorphism found in this study; however, further explorations are needed to elucidate the specific mechanisms and directionality of this interaction.

This is one of the first studies to examine both BMIz and sex as moderators for the association of leptin and bone. Other strengths include its large sample size, the racial/ethnic diversity, and the intentional oversampling 
Fig. 2. Summary of results in models examining sex by leptin interaction.

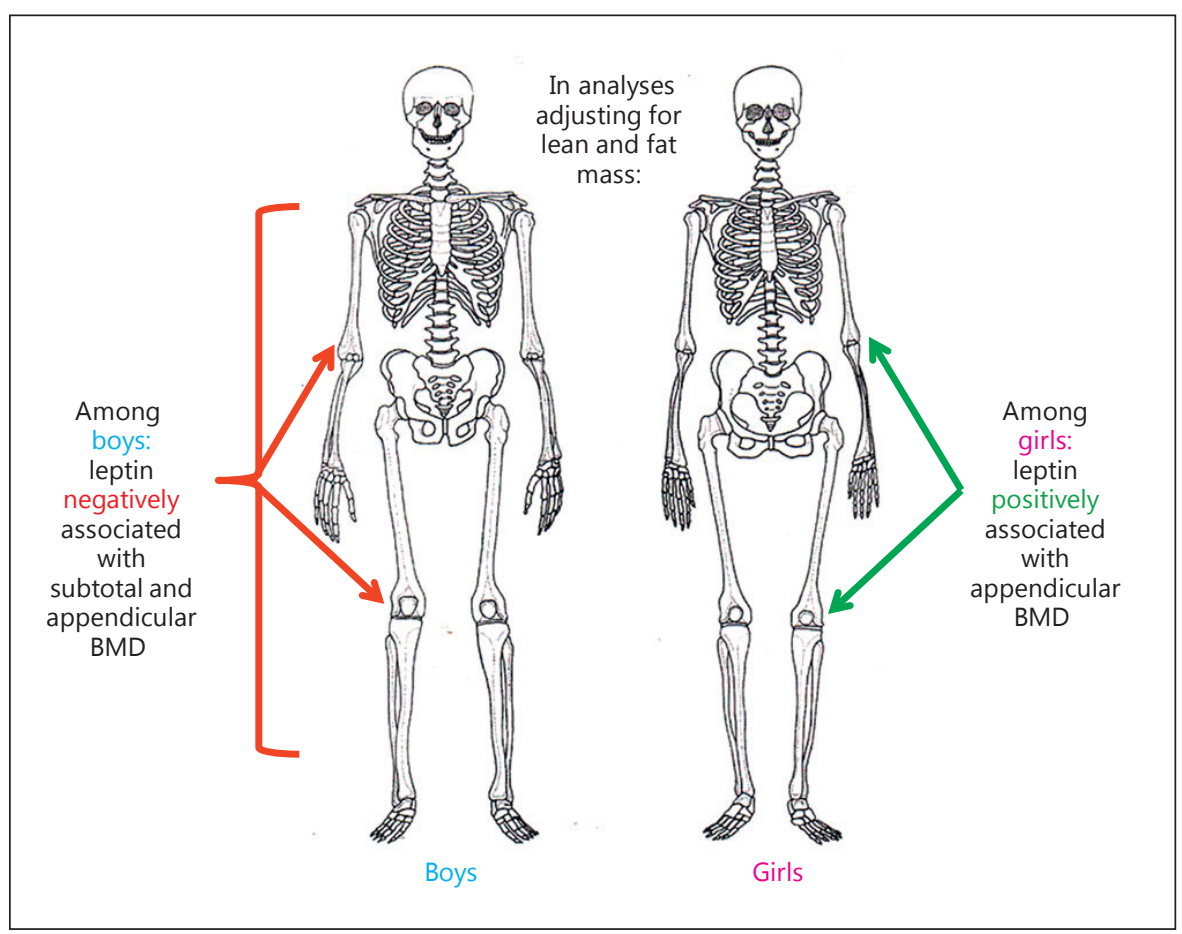

for overweight or obese children, a group that had been largely neglected in past studies examining leptin and its relation to bone composition. Given the high prevalence of obesity in children and adolescents, it is important to examine the effects of the obese hormonal environment on growth and development. However, the observational nature of the current study limits any causal conclusions. For instance, temporal associations between prolonged hyperleptinemia and changes in bone parameters cannot be captured in a cross-sectional study. Also, some of the observed differences in bone measurements might be due to confounding hormonal parameters, such as insulin, insulin-like growth factor 1, estrogen, or adiponectin, physical activity, calcium, or vitamin D levels, that were not measured or to the predominance of females in the sample. Despite our large sample size, we did not have sufficient power to evaluate how race might interact with sex and leptin concentration for prediction of bone parameters or to perform analyses where pubertal stage was matched between obese and nonobese girls and boys. Although the range for pubertal development was broad, the median for boys without obesity was prepubertal, which may have impacted some results. Since we used multiple dual-energy X-ray absorptiometry instruments, another limitation is that scans on all machines done contemporaneously for the same study participants were not

Leptin and Bone Mineral Density available to calculate true inter-machine coefficients of variation. However, our inter-instrument coefficients of variation, calculated using the same phantom, were within acceptable parameters. Similarly, although leptin interassay variability data were unavailable, the results were unaffected when controlling for assay type. Another limitation is the use of dual-energy X-ray absorptiometry measurements versus peripheral quantitative computed tomography; the latter would have provided more precise data on actual bone size and quantifiable areas of both trabecular and cortical bone. Unfortunately, because this study was done using data obtained in previous studies, these measures could not be obtained. However, our analyses accounted for the most pertinent covariates in examining the unique association between leptin and bone markers. Future longitudinal studies are needed to investigate the relationships between weight and leptin as well as between sex hormones and leptin, as they relate to bone growth and development in the pediatric population.

In conclusion, we found that leptin concentrations were negatively associated with bone parameters in youth. This relationship, however, was moderated by sex, such that the negative associations between leptin and bone development were mostly observed in boys. In contrast, girls tended to have positive correlations between leptin 
and bone measurements. Obesity as defined by BMIz did not help explain the increased fracture risk observed in obese children; other factors should therefore be explored, such as mechanical strain on bone at time of impact [36]. The mechanisms that promote leptin's catabolic versus anabolic actions also merit further study.

\section{Acknowledgements}

This work was supported by Intramural Research Program, National Institutes of Health (NIH), grant 1ZIAHD000641 (to J.A.Y.) from NICHD with supplemental funding from the National Institute for Minority Health and Health Disparities (NIMHD) and the Division of Nutrition Research Coordination (DNRC), NIH. A.M.A. was supported by the Division of Nutrition Research Coordination and the National Institute of Diabetes and Digestive and Kidney Diseases. J.A.Y. is a Commissioned Officer in the US Public Health Service, Department of Health and Human Services. S.A.A.-F. was supported by the NIH Medical Research Scholars Program, a public-private partnership supported jointly by the $\mathrm{NIH}$ and generous contributions to the Foundation for the NIH from Pfizer Inc., The Doris Duke Charitable Foundation, The
Newport Foundation, The American Association for Dental Research, The Howard Hughes Medical Institute, and the Colgate Palmolive Company, as well as other private donors. For a complete list please visit the foundation website at http://fnih.org/ what-we-do/current-education-and-training-programs/mrsp.

The funding organizations played no role in the design or conduct of the study; the collection, management, analysis, or interpretation of data; or the preparation of the manuscript.

\section{Disclosure Statement}

None of the authors report a conflict of interest for this paper.

\section{Authors' Contributions}

The first draft of the manuscript was written by Ms. ArmaizFlores, Dr. Kelly, and Dr. Yanovski. N.R.K., J.A.Y., O.A.G., A.P.D., A.M.A., S.M.B., V.S.H., C.K.P., M.T.-K., and L.B.S. contributed to data analysis. N.R.K., O.A.G., A.P.D., A.M.A., S.M.B., V.S.H., C.K.P., M.T.-K., and L.B.S. all provided critical review of and approval for the manuscript.

\section{References}

$\checkmark 1$ Ogden CL, Carroll MD, Lawman HG, Fryar CD, Kruszon-Moran D, Kit BK, Flegal KM: Trends in obesity prevalence among children and adolescents in the United States, 19881994 through 2013-2014. JAMA 2016;315: 2292-2299.

-2 Brito N, Fonseca M, Dinis I, Mirante A: Metabolic factors in obesity. J Pediatr Endocrinol Metab 2010;23:97-100.

-3 Felson DT, Zhang Y, Hannan MT, Anderson JJ: Effects of weight and body mass index on bone mineral density in men and women: the Framingham study. J Bone Miner Res 1993;8: 567-573.

- 4 Ka K, Rousseau MC, Lambert M, O'Loughlin J, Henderson M, Tremblay A, Alos N, Nicolau B: Association between lean and fat mass and indicators of bone health in prepubertal Caucasian children. Horm Res Paediatr 2013;80: 154-162.

5 Lazar-Antman MA, Leet AI: Effects of obesity on pediatric fracture care and management. J Bone Joint Surg Am 2012;94:855-861.

-6 Taylor ED, Theim KR, Mirch MC, Ghorbani S, Tanofsky-Kraff M, Adler-Wailes DC, Brady S, Reynolds JC, Calis KA, Yanovski JA: Orthopedic complications of overweight in children and adolescents. Pediatrics 2006;117: 2167-2174
7 Falorni A, Bini V, Molinari D, Papi F, Celi F, Di Stefano G, Berioli MG, Bacosi ML, Contessa G: Leptin serum levels in normal weight and obese children and adolescents: relationship with age, sex, pubertal development, body mass index and insulin. Int J Obes Relat Metab Disord 1997;21:881-890.

8 Ducy P, Amling M, Takeda S, Priemel M, Schilling AF, Beil FT, Shen J, Vinson C, Rueger JM, Karsenty G: Leptin inhibits bone formation through a hypothalamic relay: a central control of bone mass. Cell 2000;100: 197-207.

-9 Elefteriou F, Ahn JD, Takeda S, Starbuck M, Yang X, Liu X, Kondo H, Richards WG, Bannon TW, Noda M, Clement K, Vaisse C, Karsenty G: Leptin regulation of bone resorption by the sympathetic nervous system and cart. Nature 2005;434:514-520.

10 Holloway WR, Collier FM, Aitken CJ, Myers DE, Hodge JM, Malakellis M, Gough TJ, Collier GR, Nicholson GC: Leptin inhibits osteoclast generation. J Bone Miner Res 2002;17: 200-209.

11 Thomas T, Gori F, Khosla S, Jensen MD, Burguera B, Riggs BL: Leptin acts on human marrow stromal cells to enhance differentiation to osteoblasts and to inhibit differentiation to adipocytes. Endocrinology 1999;140:16301638.
12 Sabour H, Norouzi Javidan A, Latifi S, Shidfar F, Vafa MR, Emami Razavi SH, Larijani B, Heshmat R: Relationship between leptin and adiponectin concentrations in plasma and femoral and spinal bone mineral density in spinal cord-injured individuals. Spine J 2015;15:1-9.

13 Thomas T, Burguera B, Melton LJ 3rd, Atkinson EJ, O'Fallon WM, Riggs BL, Khosla S: Role of serum leptin, insulin, and estrogen levels as potential mediators of the relationship between fat mass and bone mineral density in men versus women. Bone 2001;29:114120.

14 Foo JP, Polyzos SA, Anastasilakis AD, Chou $S$, Mantzoros CS: The effect of leptin replacement on parathyroid hormone, RANKL-osteoprotegerin axis, and Wnt inhibitors in young women with hypothalamic amenorrhea. J Clin Endocrinol Metab 2014;99:E2252E2258.

15 Lorentzon M, Landin K, Mellstrom D, Ohlsson C: Leptin is a negative independent predictor of areal BMD and cortical bone size in young adult Swedish men. J Bone Miner Res 2006;21:1871-1878.

16 Afghani A, Goran MI: The interrelationships between abdominal adiposity, leptin and bone mineral content in overweight Latino children. Horm Res 2009;72:82-87. 
17 Campos RM, de Mello MT, Tock L, da Silva PL, Corgosinho FC, Carnier J, de Piano A, Sanches PL, Masquio DC, Tufik S, Damaso AR: Interaction of bone mineral density, adipokines and hormones in obese adolescents girls submitted in an interdisciplinary therapy. J Pediatr Endocrinol Metab 2013;26:663668.

$>18$ Li XP, Zeng S, Wang M, Wu XP, Liao EY: Relationships between serum omentin-1, body fat mass and bone mineral density in healthy Chinese male adults in Changsha area. J Endocrinol Invest 2014;37:991-1000.

-19 Huang KC, Cheng WC, Yen RF, Tsai KS, Tai TY, Yang WS: Lack of independent relationship between plasma adiponectin, leptin levels and bone density in nondiabetic female adolescents. Clin Endocrinol (Oxf) 2004;61: 204-208.

20 Dimitri P, Jacques RM, Paggiosi M, King D, Walsh J, Taylor ZA, Frangi AF, Bishop N, Eastell R: Leptin may play a role in bone microstructural alterations in obese children. J Clin Endocrinol Metab 2015;100:594-602.

21 Kuczmarski RJ, Ogden CL, Guo SS, Grummer-Strawn LM, Flegal KM, Mei Z, Wei R, Curtin LR, Roche AF, Johnson CL: 2000 CDC growth charts for the United States: methods and development. Vital Health Stat 2002;11: $1-190$.

-22 Crabtree NJ, Arabi A, Bachrach LK, Fewtrell M, El-Hajj Fuleihan G, Kecskemethy HH, Jaworski M, Gordon CM; International Society for Clinical Densitometry: Dual-energy X-ray absorptiometry interpretation and reporting in children and adolescents: the revised 2013 ISCD Pediatric Official Positions. J Clin Densitom 2014;17:225-242.

$>23$ Tanner JM: Growth and maturation during adolescence. Nutr Rev 1981;39:43-55.
4 Zachmann M, Prader A, Kind HP, Hafliger H, Budliger $\mathrm{H}$ : Testicular volume during adolescence. Cross-sectional and longitudinal studies. Helv Paediatr Acta 1974;29:61-72.

25 Marshall WA, Tanner JM: Variations in the pattern of pubertal changes in boys. Arch Dis Child 1970;45:13-23.

26 Fleisch AF, Agarwal N, Roberts MD, Han JC, Theim KR, Vexler A, Troendle J, Yanovski SZ, Yanovski JA: Influence of serum leptin on weight and body fat growth in children at high risk for adult obesity. J Clin Endocrinol Metab 2007;92:948-954.

27 Kruskal WH, Wallis WA: Use of ranks in onecriterion variance analysis. J Am Stat Assoc 1952;47:583-621.

28 Benjamini Y, Hochberg T: Controlling the false discovery rate: a practical and powerful approach to multiple testing. J R Stat Soc Series B Stat Methodol 1995;57:289-300.

29 Yang WH, Tsai CH, Fong YC, Huang YL, Wang SJ, Chang YS, Tang CH: Leptin induces oncostatin $\mathrm{M}$ production in osteoblasts by downregulating miR-93 through the Akt signaling pathway. Int J Mol Sci 2014;15:1577815790.

30 Moon HS, Huh JY, Dincer F, Schneider BE, Hasselgren PO, Mantzoros CS: Identification and saturable nature of signaling pathways induced by metreleptin in humans: comparative evaluation of in vivo, ex vivo, and in vitro administration. Diabetes 2015;64:828-839.

31 Milz S, Boszczyk A, Putz R: Development and functional structure of the epiphyseal plate (in German). Orthopade 2002;31:835-840.

32 Maes C, Kronenberg HM: Postnatal bone growth: growth plate biology, bone formation, and remodeling; in Glorieux FH, Pettifor JM, Jüppner H (ed): Pediatric Bone, ed 2. San Diego, Academic Press, 2012, chapt 4, pp 55-82.
3 Tsurusaki K, Ito M, Hayashi K: Differential effects of menopause and metabolic disease on trabecular and cortical bone assessed by peripheral quantitative computed tomography (pQCT). Br J Radiol 2000;73:14-22.

34 Meier DE, Orwoll ES, Jones JM: Marked disparity between trabecular and cortical bone loss with age in healthy men. Measurement by vertebral computed tomography and radial photon absorptiometry. Ann Intern Med 1984;101:605-612.

35 Whitfield JF, Morley P, Willick GE, Isaacs RJ, MacLean S, Ross V, Barbier JR, Divieti P, Bringhurst FR: Lactam formation increases receptor binding, adenylyl cyclase stimulation and bone growth stimulation by human parathyroid hormone (hPTH)(1-28) $\mathrm{NH}_{2}$. J Bone Miner Res 2000;15:964-970.

36 Moon RJ, Lim A, Farmer M, Segaran A, Clarke NM, Dennison EM, Harvey NC, Cooper C, Davies JH: Differences in childhood adiposity influence upper limb fracture site. Bone 2015;79:88-93.

$>37$ Klein KO, Baron J, Colli MJ, McDonnell DP, Cutler GB Jr: Estrogen levels in childhood determined by an ultrasensitive recombinant cell bioassay. J Clin Invest 1994;94:24752480

38 Li XF, Wang SJ, Jiang LS, Dai LY: Stage specific effect of leptin on the expressions of estrogen receptor and extracellular matrix in a model of chondrocyte differentiation. Cytokine 2013;61:876-884

39 Ohlsson C, Engdahl C, Borjesson AE, Windahl SH, Studer E, Westberg L, Eriksson E, Koskela A, Tuukkanen J, Krust A, Chambon $\mathrm{P}$, Carlsten H, Lagerquist MK: Estrogen receptor-alpha expression in neuronal cells affects bone mass. Proc Natl Acad Sci USA 2012;109:983-988.

40 Freeman EW, Sammel MD, Lin H, Gracia CR: Obesity and reproductive hormone levels in the transition to menopause. Menopause 2010;17:718-726. 